\title{
Oxidative stress in marathon runners: interest of antioxidant supplementation
}

\author{
Mari-Carmen Gomez-Cabrera ${ }^{1}$, Agustín Martínez ${ }^{2}$, Gustavo Santangelo ${ }^{2}$, Federico V. Pallardó ${ }^{2}$ Juan $_{\text {Sastre }}^{2}$ \\ and Jose Viña ${ }^{2} *$ \\ ${ }^{1}$ Catholic University of Valencia, Spain \\ ${ }^{2}$ Department of Physiology, University of Valencia, School of Medicine, Blasco Ibañez, 15, E-46010 Valencia
}

\begin{abstract}
We have recently reported that xanthine oxidase is involved in the generation of free radicals in exhaustive exercise. Allopurinol, an inhibitor of xanthine oxidase, prevents it. The aim of the present work was to elucidate the role of exercise-derived reactive oxygen species in the cell signalling pathways involved in the adaptation to exercise in man. We have found that exercise causes an increase in the activity of plasma xanthine oxidase and an activation of NF- $\mathrm{KB}$ in peripheral blood lymphocytes after marathon running. This activation is dependent on free radical formation in exercise: treatment with allopurinol completely prevents it. In animal models, we previously showed that NF- $\mathrm{BB}$ activation induced by exhaustive physical exercise leads to an increase in the expression of superoxide dismutase, an enzyme involved in antioxidant defence. We report evidence in man that reactive oxygen species act as signals in exercise as decreasing their formation prevents activation of important signalling pathways which can cause useful adaptations in cells.
\end{abstract}

NF-кB: Reactive oxygen species: Signal transduction: Marathon runners

Unaccustomed exercise increases the generation of reactive oxygen species (ROS) in biological tissues (Davies et al. 1982). We showed that these radicals cause cellular damage only when exercise is exhaustive (Sastre et al. 1992; GomezCabrera et al. 2003). We also reported that allopurinol protects against cell damage caused by exhaustive exercise both in man (Gomez-Cabrera et al. 2003) and in experimental animals (Viña et al. 2000a,b), thus highlighting the role of xanthine oxidase $(\mathrm{XO})$ in ROS generation during exercise. However, free radicals not only cause damage but also have a role in cell signalling (Reid, 2001). Animals frequently exposed to exercise (chronic training) have shown less oxidative damage after exhaustive exercise than untrained ones. This is largely attributed to the up-regulation of endogenous antioxidant enzymes such as mitochondrial Mn superoxide dismutase (MnSOD), glutathione peroxidase and $\gamma$-glutamylcysteine synthetase (Salminen et al. 1984). Since the adaptive properties result from the cumulative effects of repeated exercise bouts, the initial signal for the stimulation leading to the long-term modulation must occur after each individual exercise bout (Hollander et al. 2001). Several oxidative stress-sensitive signalling pathways are operational in mammalian systems and play an important role in maintaining cellular oxidant-antioxidant balance. One of the most important pathways that may be activated by ROS involves nuclear protein NF-кB (Baeuerle \& Baltimore, 1988). We recently showed that acute exercise activates NF- $\kappa$ B signalling pathway in rat skeletal muscle, leading to increased expression of MnSOD, an enzyme involved in antioxidant defence (Ji et al. 2004). Since MnSOD is a well-known antioxidant enzyme that modulates its gene expression in response to cellular pro-oxidative and antioxidant status, we speculate that NF- $\kappa \mathrm{B}$ activation by ROS generated during exercise may be the underlying mechanism for adaptation to training and increased expression of antioxidant enzymes.

In the present paper, we examine the role of ROS in generating signals important for the adaptation of cells to exercise in athletes after running a marathon. We report that ROS generated during marathon running induce an activation of plasma $\mathrm{XO}$, a superoxide-generating enzyme, which we have shown is essential for the generation of oxidative stress in exercise in animals. We also report an exercise-induced NF- $\kappa$ B activation in peripheral blood lymphocytes from marathon runners. Prevention of ROS formation by inhibition of XO with allopurinol abolished these effects. These results are in accordance with the data from previous animal studies in which we reported an activation of mitogen-activated protein kinases, which in turn activate $\mathrm{NF}-\kappa \mathrm{B}$ resulting in an increased expression of important cellular proteins such as inducible nitric oxide synthase, endothelial nitric oxide synthase and MnSOD. Prevention of ROS formation by inhibition of XO abolishes these effects.

The practical implication of our study is that decreasing ROS effects by antioxidants may hinder beneficial cell adaptations during exercise.

\section{Methods}

Subjects

Volunteers were recruited from participants in the 23rd Marathon of Valencia (all males, mean age 37(SD 8) years 
and with an average of 6(SD 2) marathon races completed). Runners ( $n$ 25) were randomly divided by drawing lots into two groups: placebo ( $n$ 14) and allopurinol supplemented (300 $\mathrm{mg}$ administered orally, $2 \mathrm{~h}$ before the race; $n$ 11). The pharmacokinetics of allopurinol indicates that this dose is sufficient to effectively inhibit $\mathrm{XO}$, and to treat hyperuricaemia in clinical practice (Heunks et al. 1999). Time to complete the marathon was not statistically different between the two groups of athletes.

Blood was drawn from an antecubital vein $1 \mathrm{~d}$ before and immediately after the marathon. All the runners gave informed, written consent. The experimental protocol conformed to the standards set by the Declaration of Helsinki and the procedures were approved by the Committee on Ethics in Research of the Faculty of Medicine, University of Valencia, Spain.

\section{Lymphocyte isolation and assay of $N F-\kappa B$}

Lymphocytes were isolated from fresh whole blood using Ficoll-Paque Plus (17-1440-02; Pharmacia) and low-speed centrifugation (Harris \& Ukaejiofo, 1970). The isolated suspension of lymphocytes was subjected to whole-cell protein extraction for the determination of NF-кB p50 activation, which was performed using an ELISA method according to the manufacturer's instructions (TransAM NF-кB p50 Chemi: Active Motif). Briefly, the primary antibody used to detect NF- $\kappa \mathrm{B}$ recognizes an epitope on $\mathrm{p} 50$ that is accessible only when NF-кB is activated and bound to its target DNA.

\section{Malondialdehyde determinations}

Malondialdehyde concentrations were determined in human plasma using HPLC as described by Young et al. (1987).

\section{Determination of xanthine oxidase activity}

Amplex ${ }^{\circledR}$ Red Xanthine/Xanthine Oxidase Assay Kit (Molecular Probes) was used to determine xanthine oxidase activity in human plasma as described by the manufacturer.

\section{Statistical analysis}

Results are expressed as means and standard deviations. Statistical analysis was performed by the least-significant difference test which consisted of two steps. First an ANOVA was performed. The null hypothesis was accepted for all numbers of those sets in which $F$ was non-significant at the level of $P \leq 0 \cdot 05$. Second, the sets of data in which $F$ was significant were examined by the modified $t$ test using $P \leq 0.05$ as the critical limit.

\section{Results}

Activation of plasma xanthine oxidase after marathon running

XO activity increased from 0.65 (SD 0.1 ) IU/l before exercise to 1.5 (SD 0.3) IU/l after the marathon ( $n 14, P<0.05)$. This was prevented by treatment with allopurinol $(0.90$ (SD 0.05$)$ IU/l before exercise $v .0 .97$ (SD 0.1) IU/l after marathon running, $n$ 11).

Activation of $N F-\kappa B$ after marathon running and prevention by allopurinol

Marathon running induced activation of the p50 subunit of the $\mathrm{NF}-\kappa \mathrm{B}$ complex in lymphocytes (Fig. 1). This was prevented by treatment with allopurinol.

\section{Inhibition of xanthine oxide prevents plasma lipid peroxidation in marathon running}

To determine whether $\mathrm{XO}$ is indeed responsible for oxidative stress associated with marathon running, we measured lipid peroxidation in the plasma of athletes before and after a marathon. Malondialdehyde (a measure of lipid hydroperoxide levels) was significantly increased after marathon running $(P<0 \cdot 05)$. However, this increase was absent following allopurinol treatment (Fig. 1).

\section{Discussion}

Exercise generates free radicals (Davies et al. 1982). When free radical formation overwhelms antioxidant defences,

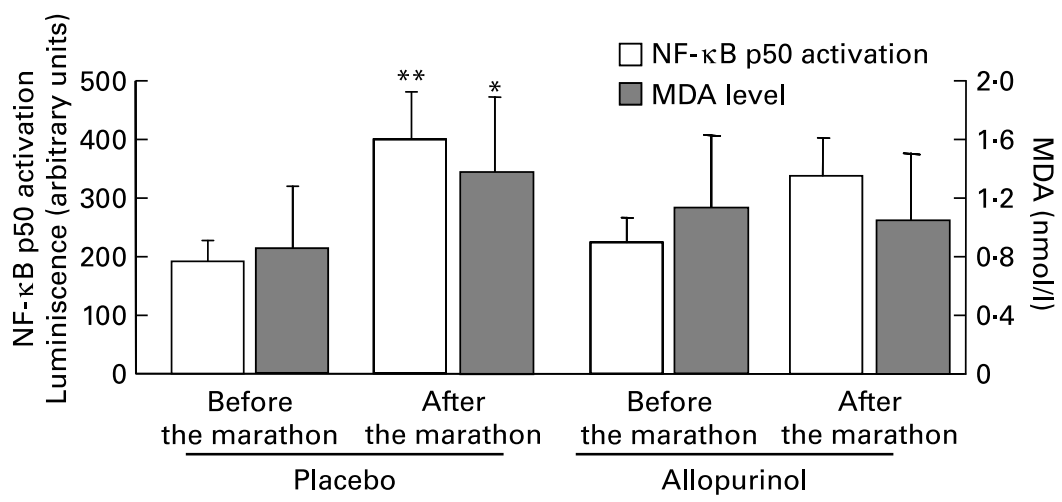

Fig. 1. NF-кB activation in lymphocytes and plasma Malondialdehyde (MDA) levels after marathon running are blocked by treatment with allopurinol. Values are means with standard deviation indicated by vertical bars for eleven subjects in the placebo group and fourteen subjects in the allopurinol-treated group. Mean values were significantly different compared with those before the marathon: ${ }^{*} P<0.05,{ }^{* \star} P<0.01$. 
oxidative stress occurs. In this context, exercise causes oxidative stress only when it is exhaustive (Sastre et al. 1992).

The mechanism of free radical formation in exercise has not been completely elucidated. We showed that XO is involved in free radical generation in exercised animals. Recently, we found that allopurinol (an inhibitor of $\mathrm{XO}$ ) prevents damage associated with exhaustive exercise in the Tour de France (Gomez-Cabrera et al. 2003).

In the current study, we show that XO-derived ROS activates an NF- $\kappa \mathrm{B}$ signalling pathway in peripheral blood lymphocytes after running a marathon.

We previously reported that inhibition of $\mathrm{XO}$ with allopurinol prevents damage associated with exhaustive exercise in animals (Viña et al. 2000a,b) and man (Gomez-Cabrera et al. 2003). We have used allopurinol to inhibit XO (Heunks et al. 1999; Viña et al. 2000a,b; Gomez-Cabrera et al. 2003). Although allopurinol may act as a chemical antioxidant, we found this is not the case at the dose used in the present study. In human subjects treated with $300 \mathrm{mg}$ allopurinol, its plasma concentration does not reach the levels required to exhibit antioxidant properties (Heunks et al. 1999).

The idea that exercise generates signals important in cell physiology has been suspected for a long time. Vider et al. (2001) first showed that physical exercise induces activation of NF-кB in human peripheral blood lymphocytes. Two years later, Khassaf et al. (2003) found that supplementation of subjects with vitamin $\mathrm{C}$ significantly diminished the ability of lymphocytes to express protective enzymes (SOD and catalase) and heat shock proteins following exposure to an exogenous oxidant. They concluded that in vitamin C-supplemented subjects, adaptive responses to oxidants were attenuated. We report, for the first time, that XO-derived ROS formed during marathon running are responsible for NF- $\mathrm{NB}$ activation because, as shown in Fig. 1, inhibiting their formation (with allopurinol) prevents NF- $\mathrm{KB}$ activation and, possibly, the subsequent up-regulation of the expression of protective, antioxidant enzymes.

In the present paper, we report that ROS produced in exercise act as signals that regulate molecular events important in cell adaptations to exercise in athletes. Thus, free radicals act as a double-edged sword: they cause oxidative damage, but they also up-regulate the expression of enzymes that are important for antioxidant defense. The practical consequence is that antioxidant administration prevents such adaptations and, thus, the recommendation of taking antioxidant supplements before exercise should be revised as they may prevent useful adaptations induced by exercise.

\section{Acknowledgements}

This work was supported by grants from CICYT (BFI-20012849 and SAF2004-03755 to J. V.; SAF2002/00885 to
F. V. P.) and from Instituto de Salud Carlos III, RCMN (C03/08), Madrid, Spain. M.-C. G.-C. is recipient of a DANONE grant for research. We thank Pilar Monforte for her excellent technical assistance and the Correcaminos Running Society for their cooperation in this work.

\section{References}

Baeuerle PA \& Baltimore D (1988) Activation of DNA binding activity in an apparently cytoplasmic precursor of the NF- $\mathrm{KB}$ transcriptional factor. Cell 53, 211-217.

Davies KJA, Quintanilha AT, Brooks GA \& Packer L (1982) Free radicals and tissue damage produced by exercise. Biochem Biophys Res Commun 107, 1198-1205.

Gomez-Cabrera MC, Pallardo FV, Sastre J, Viña J \& Garcia-del-Moral L (2003) Allopurinol and markers of muscle damage among participants in the Tour de France. JAMA 289, 2503-2504.

Harris R \& Ukaejiofo EO (1970) Tissue typing using a routine onestep lymphocyte separation procedure. $\mathrm{Br} J$ Haematol 18, 229-235.

Heunks LM, Viña J, van Herwaarden CL, Folgering HT, Gimeno A \& Dekhuijzen PN (1999) Xanthine oxidase is involved in exerciseinduced oxidative stress in chronic obstructive pulmonary disease. Am J Physiol 277, 1697-1704.

Hollander J, Fiebig R, Gore M, Ookawara T, Ohno H \& Ji LL (2001) Superoxide dismutase gene expression is activated by a single bout of exercise in rat skeletal muscle. Pflugers Arch 442, 426-434.

Ji LL, Gomez-Cabrera MC, Steinhafel N \& Viña J (2004) Acute exercise activates nuclear factor (NF) $\kappa B$ signaling pathway in rat skeletal muscle. FASEB $J$ 18, 1500-1506.

Khassaf M, McArdle A, Esanu C, et al. (2003) Effect of vitamin C supplements on antioxidant defence and stress proteins in human lymphocytes and skeletal muscle. J Physiol 549, 645-652.

Reid MB (2001) Redox modulation of skeletal muscle contraction: what we know and what we don't. J Appl Physiol 90, 724-731.

Salminen A, Kainulainen H \& Vihko V (1984) Endurance training and antioxidants of lung. Experientia 15, 822-823.

Sastre J, Asensi N, Gascó E, et al. (1992) Exhaustive physical exercise causes oxidation of glutathione status in blood: prevention by antioxidant administration. Am $J$ Physiol 32, R992-R995.

Vider J, Laaksonen DE, Kilk A, et al. (2001) Physical exercise induces activation of NF- $\mathrm{BB}$ in human peripheral blood lymphocytes. Antioxid Redox Signal 3, 1131-1137.

Viña J, Gimeno A, Sastre J, et al. (2000a) Mechanism of free radical production in exhaustive exercise in humans and rats; role of xanthine oxidase and protection by allopurinol. IUBMB Life 49, $539-544$.

Viña J, Gomez-Cabrera MC, Lloret A, et al. (2000b) Free radicals in exhaustive physical exercise: mechanism of production, and protection by antioxidants. IUBMB Life 50, 271-277.

Young SHY, Knight JA, Hopfer SM, Zaharia O, Leach CN \& Sunderman FW (1987) Lipoperoxides in plasma as measured by liquid-chromatographic separation of malondialdehyde-thiobarbituric acid adduct. Clin Chem 33, 214-220. 